\title{
Gestión de demanda en usuarios residenciales evaluando su impacto a través de un modelo de dinámica de opinión
}

\section{Demand management in residential customers with impact assess- ment via opinion dynamics}

\author{
Mateo Cortés-Guzmán ${ }^{1}$, Orlando González-Vivas ${ }^{2}$, Elkin Moreno-Soto ${ }^{3}$, Carlos Cusgüen ${ }^{4}$, Eduardo Alirio \\ Mojica-Nava ${ }^{5}$, Fabio Pavas-Martínez ${ }^{6}$ \\ ${ }^{1}$ Grupo de investigación PAAS-UN, Departamento de Ingeniería eléctrica y electrónica, Universidad Nacional de Colombia, \\ Colombia. Email: macortesgu @unal.edu.co \\ ${ }^{2}$ Grupo de investigación PAAS-UN, Departamento de Ingeniería eléctrica y electrónica, Universidad Nacional de Colombia, \\ Colombia. Email: oagonzalezv@unal.edu.co \\ ${ }^{3}$ Grupo de investigación PAAS-UN, Departamento de Ingeniería eléctrica y electrónica, Universidad Nacional de Colombia, \\ Colombia. Email: emorenoso@unal.edu.co \\ ${ }^{4}$ Grupo de investigación PAAS-UN, Departamento de Ingeniería eléctrica y electrónica, Universidad Nacional de Colombia, \\ Colombia. Email: cacusgueng@unal.edu.co \\ ${ }^{5}$ Grupo de investigación PAAS-UN, Departamento de Ingeniería eléctrica y electrónica, Universidad Nacional de Colombia, \\ Colombia. Email: eamojican@unal.edu.co \\ ${ }^{6}$ Grupo de investigación PAAS-UN, Departamento de Ingeniería eléctrica y electrónica, Universidad Nacional de Colombia, \\ Colombia. Email: fapavasm@unal.edu.co
}

RECIBIDO: Mayo 19, 2016. ACEPTAdO: Marzo 1, 2017. Versión FInAL: Mayo 15, 2017.

\section{RESUMEN}

Se propone un mecanismo de gestión de demanda que consiste en aplicar un incentivo mixto cuya primera etapa plantea un incentivo directo (financiero) enfocado en un pequeño grupo de usuarios que forman una semilla; y una segunda etapa en la que se evalúa la influencia del grupo semilla (incentivo social) sobre el grupo total de usuarios teniendo en cuenta los pesos de las opiniones atribuidas a otros agentes y la susceptibilidad al cambio de las opiniones. Se busca establecer el rango de alternativas de inversión factibles teniendo en cuenta las restricciones económicas de los usuarios y la propagación de influencia en la red de usuarios; obteniendo una relación entre consumo adicional, costo de la inversión y periodo de retorno. También se plantean casos de estudio aplicados en los cuales se evalúa el potencial de gestión sobre los usuarios, logrando un aumento cercano al 6.3\% en la potencia promedio en el caso ideal y de $2.65 \%$ cuando se asume una participación moderada de los usuarios que deciden utilizar una consola de videojuegos. De forma similar se logra un $16 \%$ de aumento del promedio de potencia en el caso ideal y de $7 \%$ suponiendo una tasa de aceptación moderada en la adquisición de un calentador eléctrico de paso.

PALABRAS CLAVE: Desplazamiento de carga, Dinámicas de opinión, Electricidad, Energía, Fin estratégico, Flexibilidad de la demanda, Gestión de demanda, Grafos, Influencia, Multiagente, Respuesta de la demanda.

\begin{abstract}
A mechanism for demand management is proposed which consists of a combined incentive with first stage direct (monetary) incentive applied to a small group of users forming a seed; and a second stage where the seed's influence on the total group is evaluated given the opinions' weights attributed to other agents and the susceptibility to change of self-opinions. The main goal is to establish the range of feasible investment alternatives using the combined incentive, having into account the user's budget constraints and the influence propagation through the user's network, thus obtaining the relationship between consumption' s increase, investment cost and payback period. We also propose practical study cases, with assessment of user's flexibility potential in their aggregated load curves, achieving an $6.8 \%$

Este artículo puede compartirse bajo la licencia CC BY-ND 4.0 y se referencia usando el siguiente formato: M.A. Cortés, O. A. González, E. Moreno, C. Cusgüen, E. Mojica-Nava, A. Pavas, "Gestión de demanda en usuarios residenciales evaluando su impacto a través de un modelo de dinámica de opinión”, UIS Ingenierías, vol. 16, no. 2, pp. 281 - 292, Julio - Diciembre 2017. Doi: https://doi.org/10.18273/revuin.v16n2-2017025
\end{abstract}


average power increase in the ideal case, and $2.65 \%$ when moderate participation is assumed with a videogames console as the new appliance. In a similar way, when the new appliance is an electric tankless water heater it is found an average power increase about $16 \%$ for the ideal case and around $7 \%$ when the model assumes moderate acceptance of the program.

KEYWORDS: Load displacement, Opinion dynamics, Electricity, Energy, Strategic end, Demand flexibility, Demand management, Graphs, Influence, Multiagent, Demand response.

\section{INTRODUCCIÓN}

Este documento presenta un estudio realizado, para observar la influencia potencial de las opiniones en los agentes consumidores de energía eléctrica, usuarios, como un método para realizar programas de gestión de demanda y los resultados obtenidos para un grupo de usuarios escogidos.

En primer lugar, se establece la conceptualización básica sobre teoría de grafos y modelos de dinámicas de opinión, por otra parte, para establecer la ganancia de los usuarios y la propagación de los incentivos, se presenta el capítulo 3, en los siguientes capítulos se muestra la relación entre las estrategias de gestión de demanda y la difusión y aplicación de incentivos, por último, se presentan los resultados sobre el grupo de usuarios escogido.

\section{GRAFOS, REDES Y DINÁMICAS DE OPINIÓN}

El uso de grafos facilita el análisis de sistemas complejos debido a las múltiples interacciones entre los elementos de una red. En sistemas multiagente, (grafo - agente) los enlaces y pesos del grafo se relacionan con las interacciones y parámetros de los agentes (red social).

La falta de información exacta sobre redes reales puede resolverse generando redes aleatorias conocidas como redes de mundo pequeño ${ }^{1}$, que conservan los valores del coeficiente de agrupamiento y distribución de grado vistos en redes reales. Un algoritmo para generación de éstas fue propuesto por Watts y Strogatz [1], y es utilizado en el presente artículo para generar redes de prueba y penetración del incentivo propuesto. Otro tipo de redes aleatorias, llamadas libres de escala, utilizando el algoritmo de Barabási y Albert [2], [3]. Redes como internet o la red de colaboración en investigaciones académicas, han sido identificadas como redes libres de escala.

Los modelos de dinámicas de opinión estudian la evolución de la influencia (opinión) entre un grupo de personas. Una revisión de los modelos más utilizados puede verse en [4], entre los que resalta el propuesto por Friedkin y Johnsen - FJ — en [5], [6] por ser uno de los pocos que ha tenido validaciones empíricas en grupos pequeños de individuos. El modelo FJ considera dos parámetros

\footnotetext{
${ }^{1}$ El término original en inglés es small-world networks.
}

principales para los agentes: la susceptibilidad de cambiar la opinión propia, y la confianza en cada opinión a la cual se está expuesto (propia o de un agente vecino) durante una interacción. Otro tipo de modelos propuesto por Hegselmann y Krause [7] incluye el concepto de intervalo de confianza: cada agente tiene en cuenta las opiniones de los otros cuando son similares a la propia, pero ignora posturas radicalmente distintas. La tolerancia a opiniones dispares depende de la amplitud del intervalo de confianza.

En el presente artículo se adopta el modelo FJ, debido a que la inclusión de los parámetros de susceptibilidad y confianza y las interacciones limitadas con vecinos imprimen un realismo y complejidad mayor a la evolución de la opinión que el cómputo de un promedio restringido a los agentes dentro de un intervalo. Por otro lado, el modelo FJ también permite la inclusión en el análisis de agentes persistentes, que forman una semilla para la propagación de la influencia en el resto de la red. Se implementa la versión mostrada por Mirtabatabaei et. al. en [8], y la actualización de las opiniones se muestra en la ecuación (1):

$$
\begin{aligned}
\sigma_{\mathrm{i}}(\mathrm{t}+1)= & \left(1-\mu_{i}\right) \sigma_{i}(0)+\mu_{i} x_{i} \sigma_{i}(t) \\
& +\mu_{i}\left(1-x_{i}\right) \sum_{j=1}^{n} W_{i j} \sigma_{j}(t)
\end{aligned}
$$

Donde, para el individuo $i$, el término $\sigma_{i}(t)$ representa la opinión en el tiempo $t, \mu_{i}$ la susceptibilidad a cambiar su opinión inicial, $x_{i}$ la confianza en las propias opiniones y $W_{i j}$ la credibilidad dada por el agente $i$ al vecino $j$ durante una interacción.

\section{INCENTIVOS SOCIALES Y PROPAGACIÓN DE LA INFLUENCIA}

En un sentido amplio, un incentivo puede ser cualquier medio de modificar el comportamiento de un individuo al cambiar los costos o beneficios que percibe [9]. Los incentivos de uso más extendido suelen ser del tipo financiero [10], como premios o penalidades. No obstante, dependiendo de la función de utilidad de los agentes, es posible que su flexibilidad a cambiar sea baja, i.e., el incentivo requerido para el cambio deseado en los agentes es más alto que el máximo disponible del organizador [11], 
[12]. Esto puede ser crítico cuando un castigo o penalización no es posible (si no se habla de una regulación obligatoria) o no es conveniente (por el malestar social ante la imposición). Como alternativa, puede pensarse en un incentivo mixto de origen social [13], [14], que motive el cambio mientras los agentes reciben una remuneración indirecta que sea de su interés subjetivo. Por ejemplo, si se piensa en un incentivo ambiental, los agentes podrían reaccionar con una reducción de consumo en horarios específicos si se plantea una siembra de árboles proporcional al ahorro total.

Otra alternativa es la que se desarrolla en el presente artículo, con la introducción de un incentivo mixto para aumentar el consumo de electricidad mediante la adición de un dispositivo nuevo en el hogar. La primera etapa plantea una inversión por parte del planeador que subsidia el valor del equipo a un pequeño grupo de la población, formando una semilla que propaga su influencia en el resto de la red social de consumidores. Dependiendo de la susceptibilidad de los agentes a cambiar su opinión y adquirir el dispositivo, y sujeto a que su capacidad financiera lo permita, el planeador puede recuperar su inversión y obtener beneficios con el consumo adicional. Los agentes semilla se caracterizan por una opinión inicial alta y una baja susceptibilidad a cambiar debido al subsidio, recomendando su uso durante las interacciones.

La decisión $C$ de adquirir el nuevo dispositivo que cada agente toma - y evoluciona según la dinámica de la opinión-, se modela como una variable aleatoria binaria, y su capacidad financiera $I$, modelada utilizando una distribución lognormal con parámetros $\left(\mu_{l g n}, \sigma_{l g n}\right)$ que representa la distribución de ingresos [15] de la población que participa en la dinámica. Esta curva puede estimarse utilizando el índice de GINI y los ingresos promedio [16], [17], con las relaciones mostradas en la Ec. (2). Con un índice de GINI igual a 0.498, calculado para Bogotá en 2015 [18] e ingresos promedio estimados en 1.37 SMMLV con base en [19], [20] se obtiene la curva de ejemplo mostrada en la Fig. 1.

$$
\begin{gathered}
\text { ingresos promedio }=\mathrm{e}^{\mu_{\lg n}+\frac{1}{2} \sigma_{l g n}^{2}} \\
G I N I_{l g n}=\operatorname{erf}\left(\frac{\sigma_{l g n}}{2}\right)
\end{gathered}
$$

Cada agente solo se incorpora al programa si ha tomado la decisión de adquirir el dispositivo y tiene la capacidad financiera $P_{c}$ para asumir el gasto $G$, de acuerdo con las ecuaciones (3) y (4).

$$
\begin{gathered}
C \sim B e\left(\sigma_{i}\right) \\
D=C \wedge P_{c} \\
P_{c}=\left\{\begin{array}{c}
1 \text { si } F_{I} I-G>0 \\
0 \text { en otro caso }
\end{array}\right.
\end{gathered}
$$

Donde $D$ es la decisión final, $F_{I}$ el factor de inversión (proporción de los ingresos mensuales que un usuario está dispuesto a gastar) e $I$ son los ingresos mensuales de acuerdo con la curva de la Fig. 1. $\sim B e($ ) es la distribución de Bernoulli.

Un mecanismo se compone de un conjunto $A$ de estrategias disponibles para los agentes, un conjunto de preferencias $\theta$, según las cuales cada usuario selecciona una estrategia, y una regla de decisión. Para el presente caso, el mecanismo $M$ que se obtiene se muestra en la ecuación (5):

$$
M=\left\{\begin{array}{c}
A=\{0,1\} \\
\theta=\sigma(\mu, W) \\
D=C \wedge P_{c}
\end{array}\right.
$$

Donde $\sigma$ es la opinión de acuerdo con la ecuación (1). $M$ se diferencia de un mecanismo económico tradicional en que la regla de decisión $D$ no requiere que todos los agentes lleguen a una sola decisión común, y la decisión final no requiere que algunos agentes sacrifiquen sus preferencias en pro de lograr un beneficio óptimo para la sociedad en conjunto, sino que es un agregado de las decisiones individuales.

\section{APLICACIÓN DE LA GESTIÓN DE DEMANDA}

El comportamiento de la demanda de energía y potencia eléctrica está iniciando un proceso de activación, debido a la concienciación de su propio consumo y por ende convirtiéndose en un agente dinámico de la energía. Así se genera la necesidad de revisar cómo se ve afectado el comportamiento de la demanda una vez son incluidas programas de respuesta de la demanda.

\subsection{Programas de respuesta de la demanda}

Las estrategias de respuesta de la demanda son diferentes alternativas que se adoptan en función de moldear el perfil de demanda de los usuarios. Actualmente se destacan varios programas de RD que buscan que las estrategias sean aplicadas con el fin de lograr diferentes acciones sobre el perfil agregado de los usuarios:

Programas RD Basados en Incentivos: Del tipo voluntario, donde los esfuerzos de los usuarios por reducir el consumo se ven recompensados a través de incentivos sobre sus facturas [12], [13].

Programas RD Basados en precios: Del tipo regulatorio donde se aplica una tarifa diferenciada para horas pico y valle a todos los usuarios quienes en la medida de sus intereses deciden reaccionar o no ante la medida. [11]. 


\subsection{Acciones}

Son los objetivos o fines estratégicos a alcanzar en la curva de demanda agregada de los usuarios, estas pueden ser individuales o combinadas y para este documento se tienen en cuenta las enfocadas a RD.

- Ahorro de energía: Busca que el agente consumidor de un sistema disminuya el consumo promedio mensual sin importar horarios. Se relaciona con el concepto de sostenibilidad, también de interés creciente [21].

- Disminución de picos: Busca reducir únicamente los picos de potencia mediante ahorro de energía, cambio tecnológico, eficiencia energética.

- Desplazamiento de carga: Este fin trasladar el consumo de potencia entre las diferentes horas del día, buscando que la curva de demanda sea uniforme en el tiempo.

- Llenado de valles: Este fin busca aumentar el uso de la energía en las horas valle aprovechando tarifas más baratas.

- Aumento de consumo: Es una acción que busca el aumento del consumo por parte de los usuarios. Su eficacia es evaluada en el presente documento ya que se considera desde la perspectiva económico de un Operador de Red.

\section{MODELAMIENTO DE MECANISMO DE RD}

Se ha propuesto un programa de respuesta de la demanda de carácter voluntario a través de incentivos. Existe una población general objetivo de $\mathrm{N}=200$ usuarios residenciales con diferentes electrodomésticos dentro de su curva de demanda, que representan posibles consumos de usuarios residenciales de la ciudad de Bogotá. Del grupo total, se seleccionó una semilla de 20 usuarios, y parámetros de la población de acuerdo con la ecuación (6).

$$
\begin{aligned}
& \mu \sim U(0.3,0.9) \\
& \mu_{\text {semilla }} \sim U(0,0.3) \\
& W_{i j} \sim U(0,1) \\
& \sigma_{\text {inicial }} \sim U(0,0.8) \\
& \sigma_{\text {inicial-semilla }}=0.9
\end{aligned}
$$

El programa es propuesto desde el punto de vista del Operador de Red, sobre la población objetivo cuyas decisiones son desarrolladas con la ayuda de dinámicas de opinión. Una vez tomadas las decisiones del grupo en general, los cambios resultantes en la curva de demanda agregada son analizados. Los incentivos propuestos se resumen en seguida[22].

\footnotetext{
${ }^{2}$ La selección de este porcentaje es arbitraria, teniendo en cuenta un nivel de gasto moderado de los usuarios, en el que no
}

\subsection{Programa de incentivos de consumo mediante au- mento de carga}

El objetivo del programa de respuesta de la demanda es el de aumentar el consumo de energía, a través de ciertos incentivos, como es la facilidad de adquirir ciertos electrodomésticos con el compromiso del usuario a integrarlos en su hogar y aumentar su nivel de confort[22], [23]:

- Consola de videojuegos (CV): Presenta una potencia $170 \mathrm{~W}$ con un promedio uso de 2 horas diarias todos los días.

- Calentador de paso eléctrico (CP): Con potencia de $12 \mathrm{~kW}$ el cual reemplaza la ducha eléctrica en caso de ya estar presente en el hogar del usuario con promedio de uso de 30 minutos diarios.

\subsection{Aplicación de dinámicas de opinión}

El modelo de dinámicas de opinión propuesto revisa el proceso de propagación de la información, la influencia y situación socioeconómica de los usuarios para el proceso de toma de decisiones [14], [15], [16].

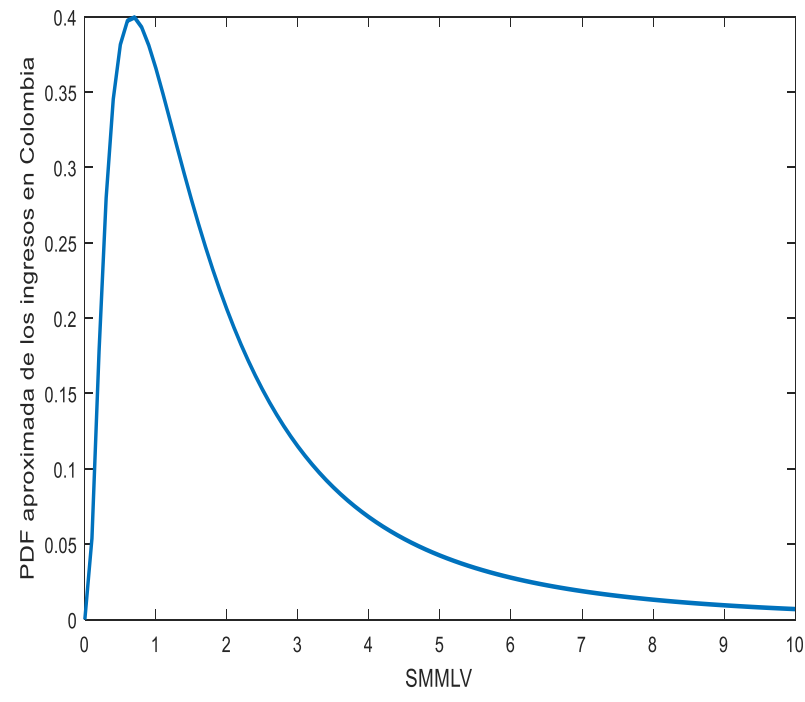

Figura 1. Distribución lognormal de los ingresos en Colombia, $\operatorname{con} \mu_{l g n}=0.5$ y $\sigma_{l g n}=0.9494$. Fuente. Elaboración propia.

Se modela un grupo de usuarios conservadores, que solo realizan la inversión si esta no afecta de manera significativa sus ingresos por tanto el factor de inversión se modeló como un $33 \%{ }^{2}$. Inicialmente se desea conocer la decisión de la población y la proporción que se acoge al plan de incentivos iniciado con el subsidio a la semilla. Para esto, se realizó un barrido Monte Carlo con T $=500$

incurrirían en deudas ni verían un impacto prolongado (de varios meses) en sus ingresos. 
iteraciones para varios costos del dispositivo, y se calcularon los valores para tres indicadores de influencia media, mostrados en la Tabla 1. Dichos indicadores se describen a continuación:

- Tienen en cuenta la proporción de agentes que se acogen al incentivo del total, o del total de elegibles (aquellos que podrían comprar el dispositivo si lo desean), y la relación entre los que lo compran entre los elegibles.

- El indicador de aptitud depende de la curva de ingresos para los agentes mostrada en la Fig. 1, y no se ve afectada por la ubicación de los agentes semilla.

- La forma de distribución de la semilla es aleatoria dentro de la población la evolución de la influencia en el resto del grupo, y es diferente al caso en el que se encuentra agrupada (Fig. 2)

- Tanto los valores de MI como los del indicador MRI son más altos en el caso de los agentes agrupados; aunque la diferencia no es grande, esto puede deberse a una mayor estabilidad de la opinión del grupo semilla dentro de la red. Para casos en los que los agentes tiendan a ser más susceptibles o más persistentes con su opinión, la influencia media de la semilla en la red cambiará de manera consecuente, según la Ec. (1).

Tabla 1. Indicadores de influencia en el modelo utilizado

\begin{tabular}{|l|c|}
\hline \multicolumn{1}{|c|}{ Indicador } & \multicolumn{1}{c|}{ Definición } \\
\hline Influencia (MI) & $\frac{1}{T} \sum_{i=1}^{T} \frac{\text { Total colaboradores }}{N}$ \\
\hline Aptitud (MA) & $\frac{1}{T} \sum_{i=1}^{T} \frac{\text { Total elegibles }}{N}$ \\
\hline $\begin{array}{l}\text { Influencia Restringida } \\
\text { (MRI) }\end{array}$ & $\frac{1}{T} \sum_{i=1}^{T} \frac{\text { Total colaboradores }}{\text { Total elegibles }}$ \\
\hline
\end{tabular}

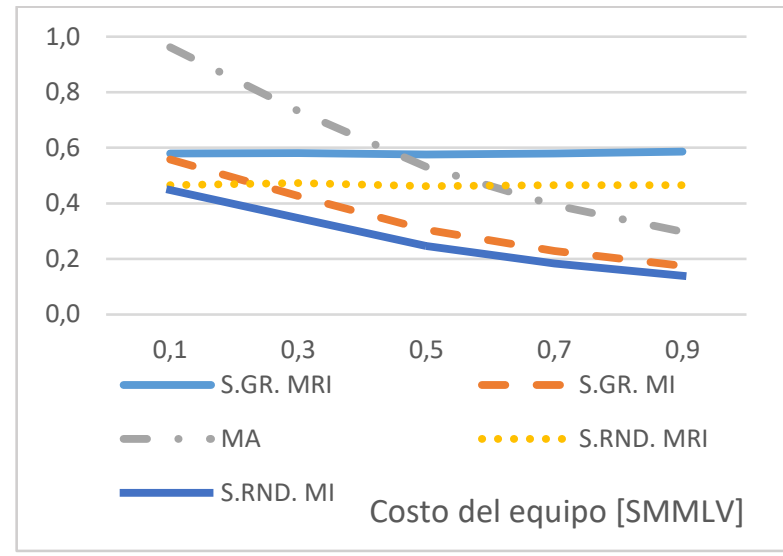

Figura 2. Valor de los indicadores de la Tabla 1, que muestra el efecto de la ubicación de los agentes semilla en la red. Fuente. Elaboración propia.

Teniendo en cuenta que la decisión final de los usuarios depende tanto de su capacidad financiera como de sus parámetros de influencia social, se realizó un barrido modificando el costo del equipo adicional que comprarían los usuarios y el costo del consumo mensual de energía asociado a dicho aparato, para obtener un mapa que caracteriza la factibilidad de que con unos parámetros sociales particulares, sea viable la introducción de un nuevo dispositivo teniendo en cuenta una inversión inicial (equipos recibidos por los agentes semilla), y las ganancias ocasionadas por el consumo adicional de los usuarios que finalmente se acojan a la campaña. La evolución para una de las iteraciones se muestra en la Fig. 3, en la que se ve que los agentes alcanzan un estado estable con rapidez, aproximadamente en 30 interacciones.

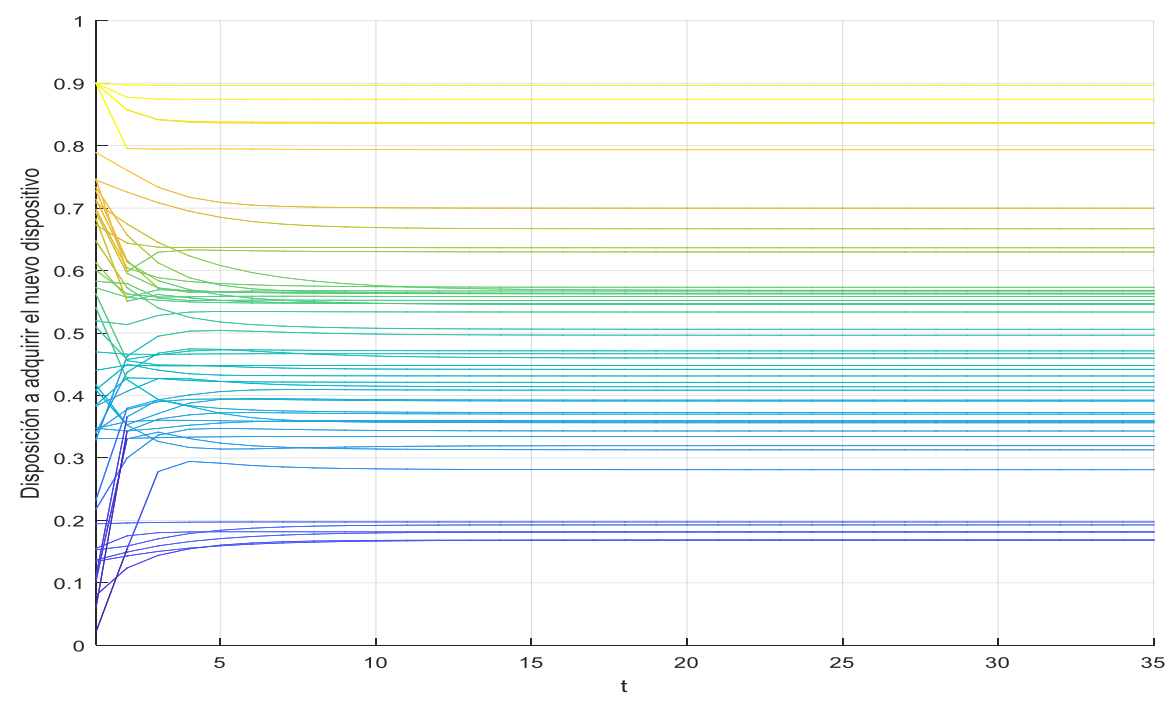

Figura 3. Evolución de la opinión para un caso con los parámetros descritos. Fuente. Elaboración propia. 
Las curvas de nivel resultantes del barrido pueden verse en la Fig. 4 donde las dos primeras líneas de contorno representan los límites de retorno de inversión (payback) de 5 y 10 meses respectivamente. La última línea amarilla comprende periodos de retorno superiores a 24 meses, que ya no serían atractivos financieramente. En este caso, si el consumo del nuevo dispositivo aporta $\$ 10000$ COP mensuales a la factura, la parte interesada solamente verá un retorno de inversión en 10 meses si el equipo cuesta alrededor de $\$ 300.000$ COP.

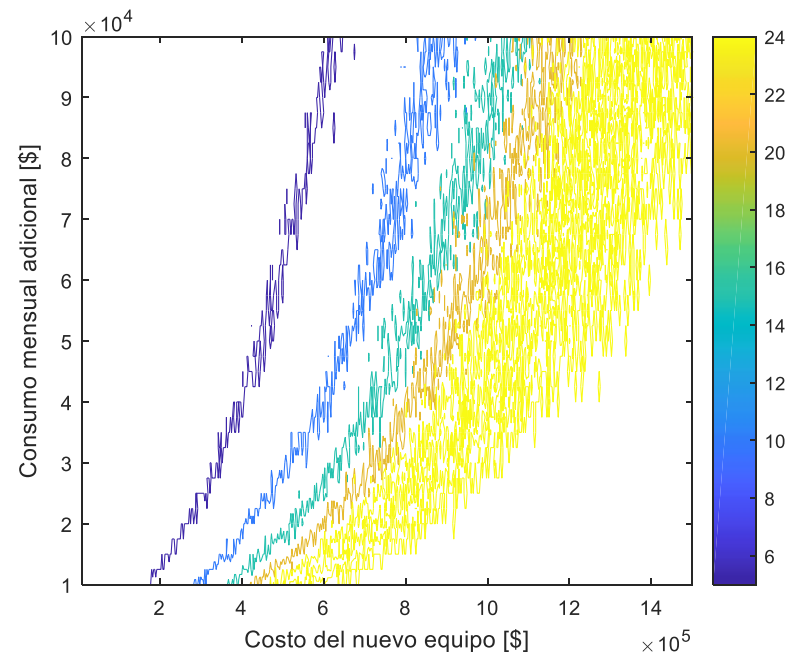

Figura 4. Periodos de retorno de la inversión dependiendo del costo del nuevo equipo y su consumo mensual medio de electricidad. Fuente. Elaboración propia.
Si el consumo se eleva a $\$ 50.000$ COP mensuales, el subsidio podría aumentar a equipos que cuesten alrededor de $\$ 700.000 \mathrm{COP}$, punto en el cual podrían ubicarse algunos modelos de calentadores de agua eléctricos. Las curvas mostradas dan una idea de los límites entre los cuales, para una población con las características descritas, sería rentable la aplicación de un incentivo mixto basado en subsidios e influencia social.

\section{RESULTADOS}

En seguida se resumen los resultados obtenidos una vez se conocen las decisiones desde la aplicación del modelo de dinámicas de opinión propuesto y reflejados en el modelamiento de la demanda con y sin el programa de $\mathrm{RD}[22]$.

\subsection{Uso de consola de videojuegos}

La consola de videojuegos en el primer escenario que es el ideal (200 usuarios aceptan mecanismo), presentaría un aumento en el promedio de consumo de los usuarios de $6.25 \%$ sobre su consumo base de referencia, en el primer escenario donde todos los 200 usuarios modelados adquieren el electrodoméstico.

En el segundo escenario el análisis de las dinámicas de opinión arrojó un total de 68 usuarios (34\%) que se acogen al programa de incentivo, adquieren el equipo y representan un aumento de $2.65 \%$ respecto de su consumo base de referencia Fig. 5.

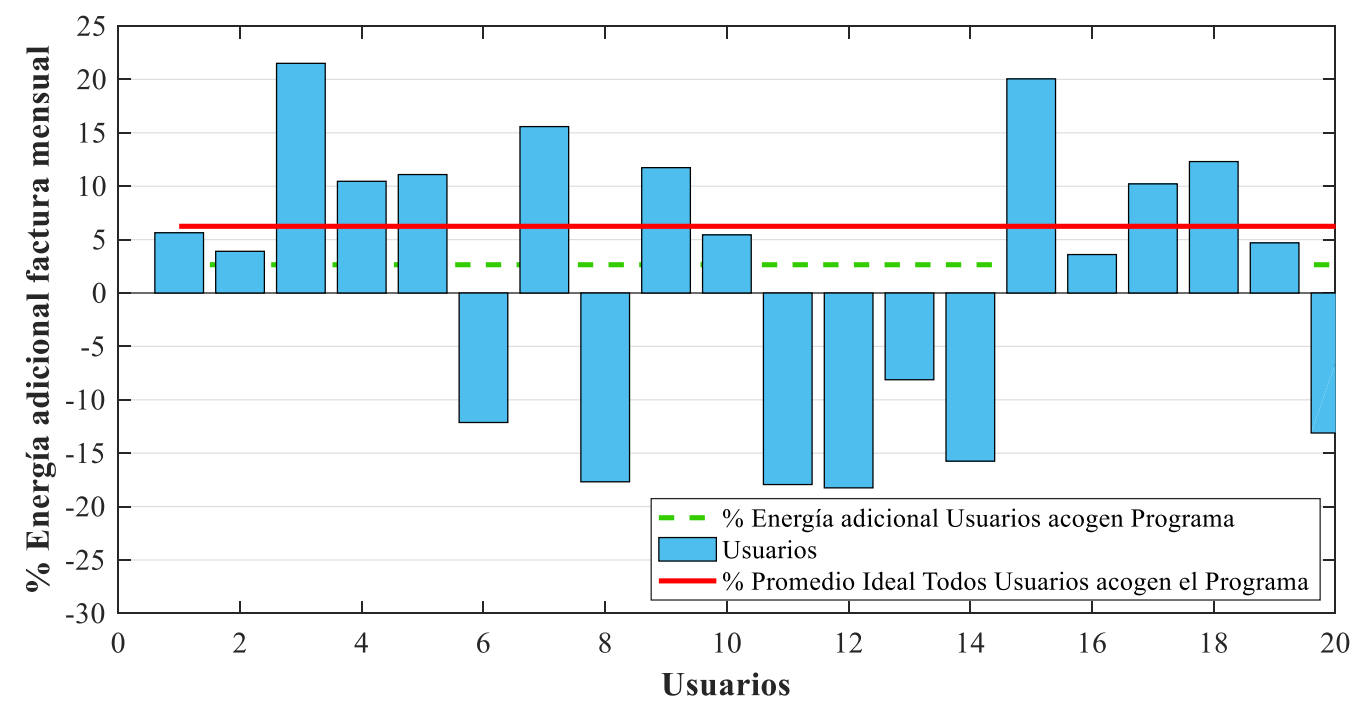

Figura 5. Porcentaje de consumo adicional CV de los 200 usuarios (Ventana 20 usuarios). Fuente. Elaboración Propia. 


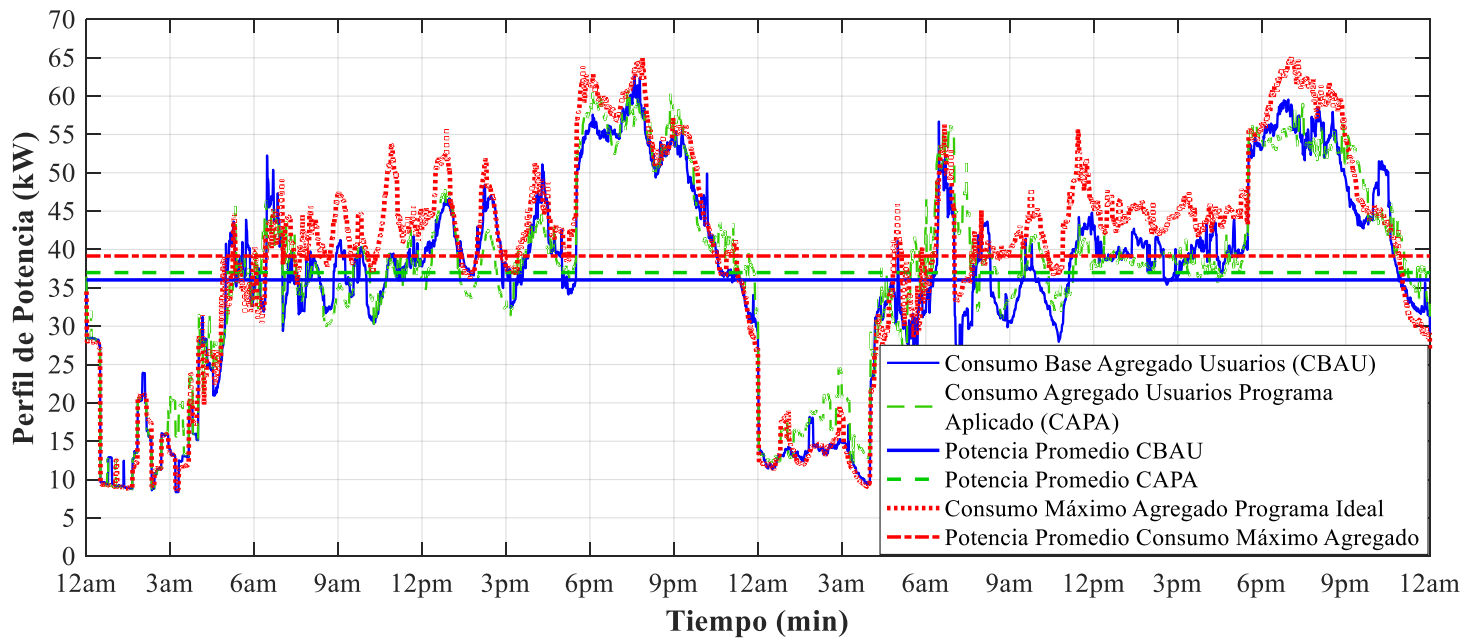

Figura 6. Perfil de potencia agregado semanal de los usuarios (ventana 2 días). Fuente. Elaboración propia.

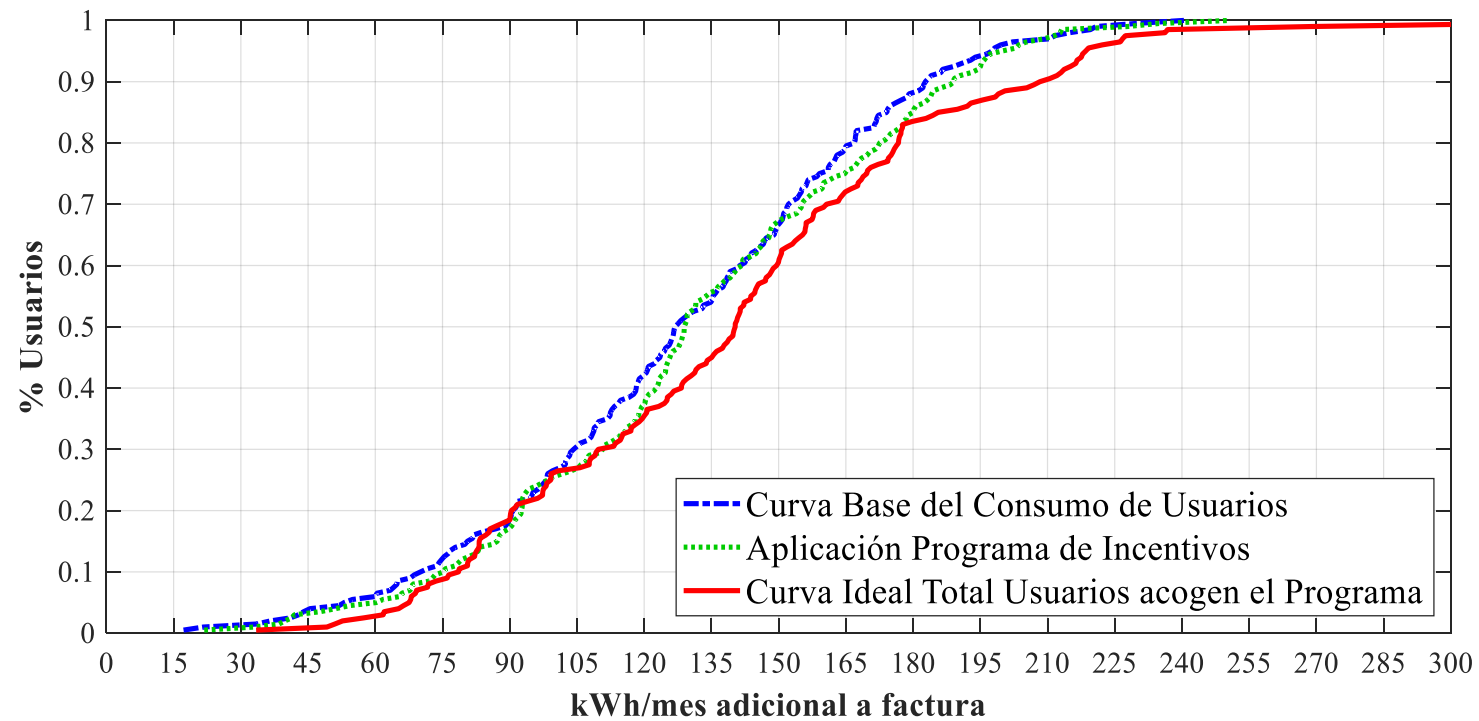

Figura 7. Función de probabilidad-CV. Fuente. Elaboración propia.

En la Fig. 6 el escenario de aplicación del programa de respuesta de la demanda arrojó un aumento en los picos de potencia promedio semanal del 35.4 - $37.2 \mathrm{~kW}$ (5.1\%), comparado con el escenario sin incentivos en el consumo agregado de los usuarios modelados. Durante la semana se presenta un pico máximo de potencia de 65 $\mathrm{kW}$ por parte de los usuarios en la curva del programa ideal, $65 \mathrm{~kW}$ cuando se genera la dinámica de opinión y $60 \mathrm{~kW}$ para el caso base de consumo agregado.

La función de probabilidad del consumo, ver Fig. 7, muestra los estadísticos resultantes (Tabla 3). El aumento en consumo de los usuarios alcanza un máximo de 12 $\mathrm{kWh} / \mathrm{mes}$ entre las curvas base e ideal para el $80 \%$ de los usuarios; lo que equivale a un máximo aumento del $10 \%$ del consumo mensual base (130 kWh/mes).
La aplicación del programa de respuesta de la demanda evaluado por la dinámica de opinión refleja un aumento máximo de $7 \mathrm{kWh} /$ mes para un $50 \%$ de los usuarios; lo cual es equivalente a un alza de $5.4 \%$ en el consumo de energía mensual base.

Tabla 2. Resumen datos función de probabilidad.

\begin{tabular}{|c|c|c|c|}
\hline \%Usuarioslaplicación & Base & $\begin{array}{c}\text { Max } \\
\text { ideal }\end{array}$ & $\begin{array}{c}\text { Dinámica } \\
\text { de opinión }\end{array}$ \\
\hline 20 & 90 & 101 & 94 \\
\hline 50 & 124 & 135 & 131 \\
\hline 80 & 169 & 182 & 169 \\
\hline
\end{tabular}

El programa de respuesta de la demanda ofrece cuando es aplicado, un rango de consumo entre 94 y 172 $\mathrm{kWh} / \mathrm{mes}$ para el $60 \%$ de los usuarios. 


\subsection{Uso de calentador de paso}

El calentador de paso en el primer escenario, presentaría un aumento en el promedio de consumo de los usuarios de $8.36 \%$ sobre su consumo base de referencia, en el primer escenario donde todos los 200 usuarios modelados adquieren el electrodoméstico.
En el segundo escenario el análisis de las dinámicas de opinión arrojó un total de 72 usuarios (36\%) que se acogen al programa de incentivo, adquieren el equipo y representan un aumento de $4.1 \%$ respecto de su consumo base de referencia Fig. 8.

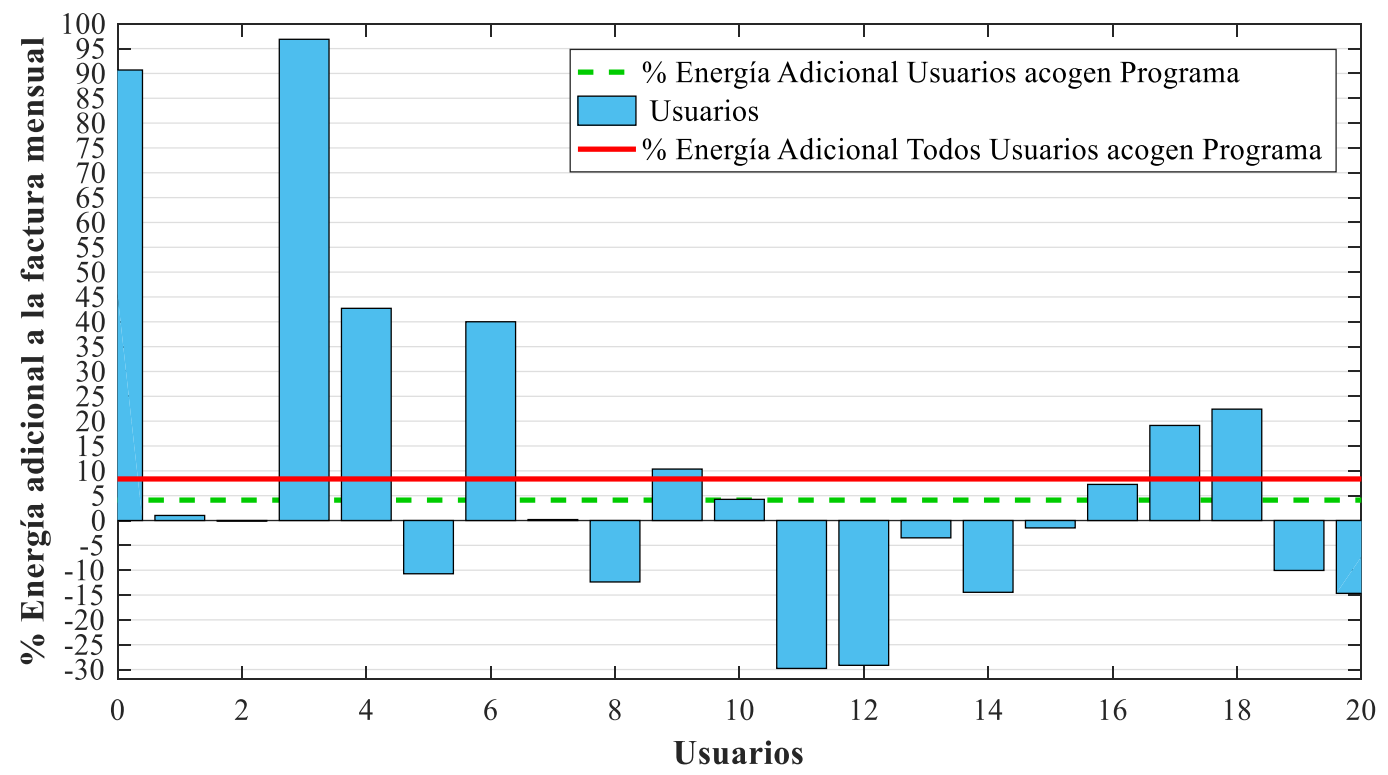

Figura 8. Porcentaje de consumo adicional CP de los 200 usuarios (Ventana 20 usuarios). Fuente. Elaboración Propia.

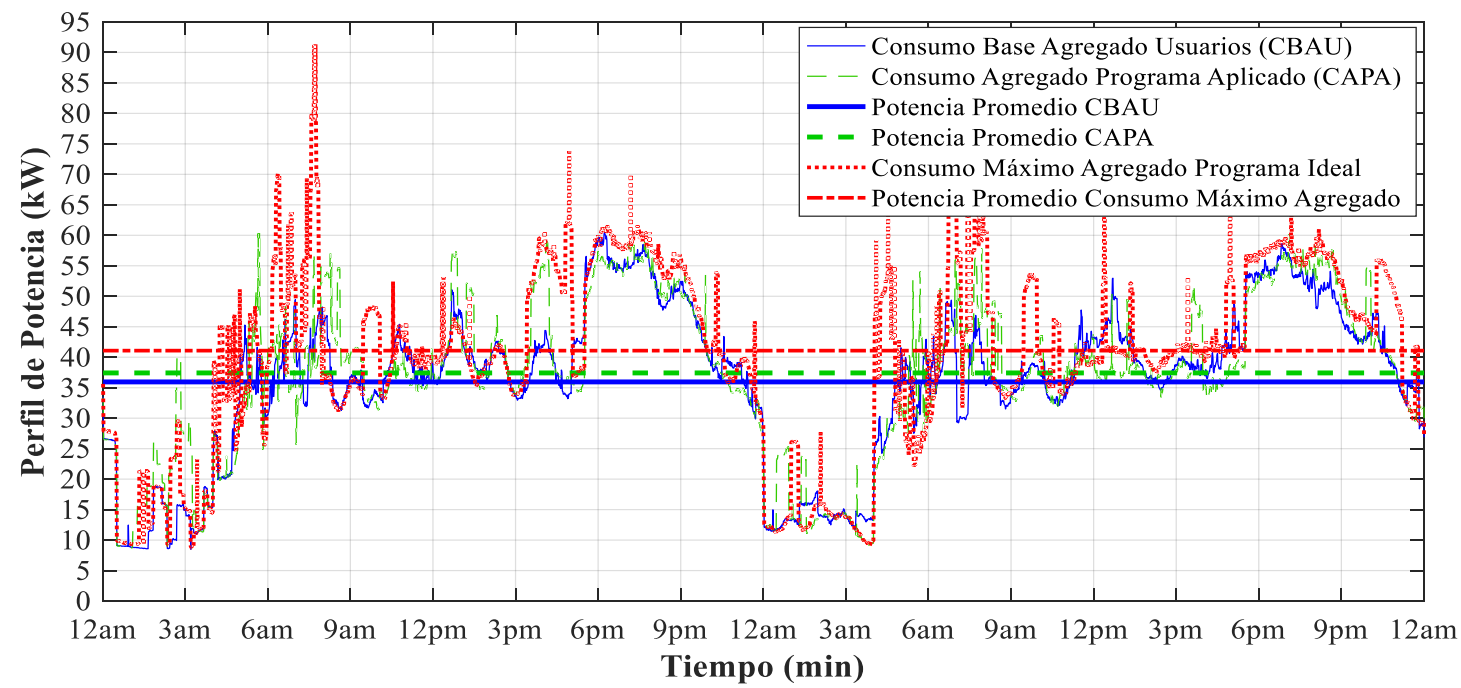

Figura 9. Perfil de potencia agregado semanal de los usuarios (ventana 2 días). Fuente. Elaboración propia. 


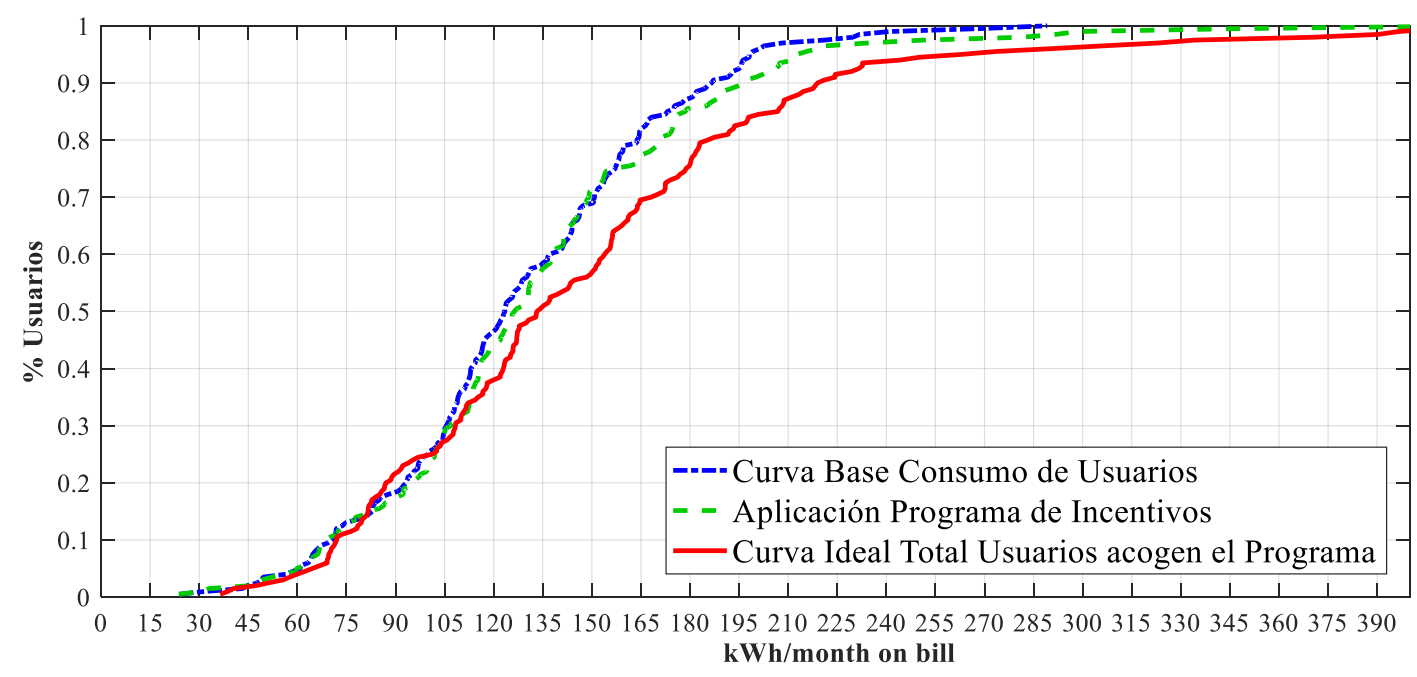

Figura 10. Función de probabilidad-CP. Fuente. Elaboración propia.

En la Fig. 9 el escenario de aplicación del programa de respuesta de la demanda arrojó un aumento en los picos de potencia promedio semanal del $35.4-38.2 \mathrm{~kW}$ (7.9\%), comparado con el escenario sin incentivos en el consumo agregado de los usuarios modelados. Durante la semana se presenta un pico máximo de potencia de 92 $\mathrm{kW}$ por parte de los usuarios en la curva del programa ideal (200 usuarios con los electrodomésticos), $60 \mathrm{~kW}$ cuando se genera la dinámica de opinión y en el caso base de consumo agregado.

La función de probabilidad del consumo en la Fig. 10, muestra los estadísticos resultantes (Tabla 4). El aumento en consumo de los usuarios alcanza un máximo de 18 $\mathrm{kWh} / \mathrm{mes}$ entre las curvas base e ideal para el $80 \%$ de los usuarios; lo que equivale a un máximo aumento del $13.8 \%$ del consumo mensual base $(130 \mathrm{kWh} / \mathrm{mes})$.

La aplicación del programa de respuesta de la demanda evaluado por la dinámica de opinión refleja un aumento máximo de $5 \mathrm{kWh} / \mathrm{mes}$ para un $80 \%$ de los usuarios; lo cual es equivalente a un alza de $3.8 \%$ en el consumo de energía mensual base.

Tabla 4. Resumen datos función de probabilidad.

\begin{tabular}{|c|c|c|c|}
\hline \%Usuarioslaplicación & Base & $\begin{array}{c}\text { Max } \\
\text { ideal }\end{array}$ & $\begin{array}{c}\text { Dinámica } \\
\text { de opinión }\end{array}$ \\
\hline 20 & 90 & 97 & 94 \\
\hline 50 & 124 & 135 & 127 \\
\hline 80 & 169 & 187 & 174 \\
\hline
\end{tabular}

El programa de respuesta de la demanda ofrece cuando es aplicado, un rango de consumo entre 94 y 174 $\mathrm{kWh} / \mathrm{mes}$ para el $60 \%$ de los usuarios.

\section{CONCLUSIONES}

\subsection{Análisis proporción de la flexibilidad}

En existe un margen de gestión de demanda el cual nos identifica la flexibilidad (entre caso ideal y base) que tendría el agente consumidor respecto a la influencia de las dinámicas de opinión, el cual no supera el 10\% (calentador) y $4 \%$ (consola) de aumento en energía promedio mensual para los dos incentivos propuestos.

En las Fig. 7 y 10, se presenta entre las funciones de probabilidad un margen muy pequeño de flexibilidad de los usuarios debido a la falta de combinación de varios elementos dentro de los incentivos, o mayor o menor disposición de uso de los dispositivos.

\subsection{Margen de reducción de picos}

Grande o pequeño existe un impacto de los programas y acciones sobre los picos de demanda (Fig. 6 y 9), elementos como la consola (potencia menor) aumenta los picos en intervalos de tiempo más largos, conservando el perfil de consumo del sistema, y otros elementos (calentador de paso) alteran considerablemente los picos de potencia y cantidad de energía consumida durante corto tiempo.

\subsection{Costo beneficio del usuario}

Teniendo en cuenta la inflexibilidad de los usuarios residenciales ante incentivos puramente financieros, una alternativa complementaria de incentivos sociales (mixto) puede tener un desempeño superior cuando la semilla está agrupada al formar un cluster con la misma opinión que tiene mayor influencia en el resto de agentes. 
De acuerdo a lo mostrado, esta puede ser alrededor de un $10 \%$ mayor.

La caracterización efectiva de los parámetros de los agentes cuyo comportamiento se modele utilizando dinámicas de opinión, sigue siendo la mayor dificultad para obtener resultados y previsiones más exactas. Sin conocer los valores reales, el análisis de escenarios permite reconocer los límites dentro de los cuales se espera que ocurra el comportamiento real, teniendo en cuenta la persistencia y la confianza en las opiniones de otros para cada agente.

\section{AGRADECIMIENTOS}

Los autores agradecen a Colciencias por el financiamiento parcial de este trabajo, enmarcado en la convocatoria No. 706-2015 "Convocatoria Nacional Jóvenes Investigadores e Innovadores año 2015".

Por otra parte, los autores agradecen al fondo de regalías por el financiamiento parcial de este trabajo, en el marco del proyecto" Incremento en las estrategias sustentables en el uso del recurso de energía eléctrica para la población vulnerable del departamento de Cundinamarca".

\section{REFERENCIAS}

[1] M. E. J. Newman, D. . J. Watts, and S. H. Strogatz, "Random graph models of social networks.," Pnas, vol. 99 Suppl 1, pp. 2566-72, 2002.

[2] R. Albert and A.-L. Barabási, "Statistical mechanics of complex networks," Rev. Mod. Phys., vol. 74, no. 1, pp. 47-97, Jan. 2002.

[3] A.-L. Barabási and E. Bonabeau, "Scale-Free Networks," Sci. Am., no. May, pp. 50-59, 2003.

[4] A. V. Proskurnikov and R. Tempo, "A Tutorial on Modeling and Analysis of Dynamic Social Networks. Part I," Annu. Rev. Control, vol. 43, pp. 65-79, 2017.

[5] N. E. Friedkin and E. C. Johnsen, "Social Influence Networks and Opinion Change," Adv. Gr. Process., vol. 16, pp. 1-29, 1999.

[6] N. E. Friedkin, "A formal theory of social power," The Journal of Mathematical Sociology, vol. 12, no. 2. pp. 103-126, 1986.

[7] R. Hegselmann and U. Krause, "Opinion Dynamics and Bounded Confidence," Simulation, vol. 5, no. 3, p. 2, 2002.

[8] A. Mirtabatabaei, P. Jia, N. E. Friedkin, and F.
Bullo, "On the reflected appraisals dynamics of influence networks with stubborn agents," Proc. Am. Control Conf., pp. 3978-3983, 2014.

[9] N. G. Mankiw, Principios de economía, 2nd ed. Madrid: McGraw Hill Interamericana de España, 2002.

[10] N. Nisan, T. Roughgarden, E. Tardos, and V. V. Vazirani, Eds., Algorithmic Game Theory. Cambridge: Cambridge University Press, 2007.

[11] M. A. Cortés Guzmán, "Diseño de un mecanismo para la gestión de la demanda de energía eléctrica aplicando control cooperativo y sistemas multi-agente," Universidad Nacional de Colombia, Departamento de Ingeniería Eléctrica y Electrónica (en curso, no publicada), 2017.

[12] Unidad de Planeación Minero Energética-UPME, "Proyección de la demanda de energía eléctrica y potencia máxima en Colombia." Rev. Junio de 2016, Bogotá, pp. 25-31, 2016.

[13] H. Gintis, S. Bowles, R. Boyd, and E. Fehr, Moral sentiments and material interests: The foundations of cooperation in economic life. MIT Press, 2005.

[14] M. A. Cortés Guzmán and E. Mojica-Nava, "Mechanism design for demand response programs with financial and non-monetary, social, incentives," Unpubl. Draft, pp. 1-8, 2017.

[15] F. Clementi and M. Gallegati, "Pareto's Law of Income Distribution: Evidence for Germany, the United Kingdom, and the United States," in Econophysics of Wealth Distributions: Econophys-Kolkata I, A. Chatterjee, S. Yarlagadda, and B. K. Chakrabarti, Eds. Milano: Springer Milan, 2005, pp. 3-14.

[16] E. L. Crow and K. Shimizu, Lognormal distributions: Theory and applications (Vol. 88). New York: M. Dekker, 1988.

[17] N. L. Johnson, S. Kotz, and N. Balakrishnan, Continuous univariate distributions Vol. 1, 2nd ed. Wiley, 1994.

[18] Observatorio de desarrollo económico, "Pobreza y desigualdad en Bogotá: resultados en los últimos años," 2016-04-14. [Online]. Available: http://observatorio.desarrolloeconomico.gov.co/base/lec torpublic.php?id=838.

[19] Departamento Administrativo Nacional de Estadística (DANE), "Ingresos y gastos de los hogares," 2007. [Online]. Available: 
http://www.dane.gov.co/index.php/estadisticas-por-

tema/pobreza-y-condiciones-de-vida/ingresos-y-gastos-

de-los-hogares. [Accessed: 28-Sep-2017].

[20] FinanzasPersonales.co, “¿Cuánto ganan en promedio los colombianos?" [Online]. Available: http://www.finanzaspersonales.co/trabajo-yeducacion/articulo/cuanto-ganan-promediocolombianos/48375. [Accessed: 28-Sep-2017].

[21] G. Osma Pinto and G. Ordoñéz Plata, "Desarrollo sostenible en edificaciones," Rev. UIS Ing., vol. 9, no. 1, Jun, 2010.

[22] O. A. González, A. Pavas, S. Sánchez, Andrés Pavas, and Samir Sánchez, "Cuantificación del Ahorro de Energía Eléctrica en Clientes Residenciales Mediante Acciones de Gestión de Demanda," UIS Ing., vol. 10, no. 2, pp. 1-8, Jul - Dic, 2017.

[23] E. Moreno, O. A. Gonzalez, A. Pavas, and A. Moreno, Elkin;González, Orlando A; Pavas, "Demand Flexibility Assessment for Residential Customers," in IEEE Workshop on Power Electronics and Power Quality Applications - PEPQA, 2017, p. 5. 